Evaluation of abiotic stress resistance in mutated populations of cauliflower (Brassica oleracea var. Botrytis)

Fuller, MP::0000-0003-3461-9728

http://hdl.handle.net/10026.1/1433

10.1007/s11240-006-9112-4

Plant Cell Tissue and Organ Culture

Springer Science and Business Media LLC

All content in PEARL is protected by copyright law. Author manuscripts are made available in accordance with publisher policies. Please cite only the published version using the details provided on the item record or document. In the absence of an open licence (e.g. Creative Commons), permissions for further reuse of content should be sought from the publisher or author. 


\title{
Evaluation of abiotic stress resistance in mutated populations of cauliflower (Brassica oleracea var. botrytis)
}

\author{
M. P. Fuller · E. M. R. Metwali · M. H. Eed • \\ A. J. Jellings
}

Received: 19 February 2006/Accepted: 14 April 2006/Published online: 7 July 2006

(C) Springer Science+Business Media B.V. 2006

\begin{abstract}
An efficient in vitro screening method has been developed for cauliflower to create NEU and NMU induced mutant lines selected on hydroxyproline containing medium. Mutant lines and control plants were sub-cultured many times on maintenance medium and stored at $5^{\circ} \mathrm{C}$ for 2 years and then tested for salt and hydroxyproline resistance as in vitro and in vivo plants. In vitro shoot tips were also sub-cultured to media containing hydroxyproline and $\mathrm{NaCl}$ for 28 days and then assessed for their leaf proline content. Non-acclimated and acclimated in vivo plants were also assessed for resistance to freezing. Populations of control and selected lines were created by mass pollination and subsequently tested for their $\mathrm{NaCl}$ and frost resistance. Control plants had little or no $\mathrm{NaCl}$ or hydroxyproline resistance whilst selected plants showed varying degrees of resistance. In vitro and in vivo responses of selected lines were correlated. Leaf proline content was increased markedly in the mutant lines and the
\end{abstract}

M. P. Fuller $(\bowtie) \cdot$ E. M. R. Metwali · A. J. Jellings

School of Biological Sciences, University of

Plymouth, Drake Circus, Plymouth PL4 8AA, UK

e-mail: mfuller@plymouth.ac.uk

M. H. Eed · E. M. R. Metwali

University of the Suez Canal, Ismailia, Egypt greatest proline contents occurred following $\mathrm{NaCl}$ stress with the most respondent line having 100-fold levels compared to the controls. Both non-acclimated and acclimated selected lines showed improved frost resistance over controls. Improvements in frost resistance were heritable but improvements in $\mathrm{NaCl}$ resistance were not. The results clearly demonstrated that $\mathrm{NaCl}$, frost and hydroxyproline resistance were stable traits over repeated in vitro sub-cultures and prolonged low temperature storage. A complete range of mutants with single, double or triple resistance traits were produced. The level of resistance however was not necessarily correlated with the level of proline and some lines showed resistance without elevated proline. It is concluded that elevated proline is not essential for improved resistance to abiotic stress in cauliflower, but where it does occur it does improve resistance.

Keywords $\mathrm{NaCl} \cdot$ Hydroxyproline $\cdot$ Frost $\cdot$ Proline $\cdot$ Mutant $\cdot$ Cauliflower $\cdot$ In vitro $\cdot$ Brassica oleracea L.
Abbreviations
IBA Indole butyric acid
RC\% Relative conductivity percentage
M\&S Murashige and Skoog (1962) 


\section{Introduction}

Abiotic environmental stresses particularly salt, freezing and drought are major limitations for plant growth and crop production worldwide. Soil salinity is principally regarded as an inherent problem of irrigated agriculture, restricting crop yield and sustained production in sizeable parts of the world. Munns and Rawson (1999) indicated that $5 \%$ (77 million ha) of the 1.5 billion ha of cultivated land is affected by salt. Wang et al. (2001) predicted that increasing salinization of arable land will have devastating effects resulting in $3 \%$ land loss within the next 25 years and up to $50 \%$ by the year 2050 . Increased salt tolerance in crops is widely recognized as an effective way to overcome the limitations to production in saline areas (Epstein 1985). In the case of economically important plants, frost can cause significant losses to crop production and reliable protection of crops from frost damage has always been an elusive goal. Frost stress is intimately linked with drought and salt stress (Guy 2003).

Breeding for tolerance against cold and salinity by classical methods of selection and crossing is a time consuming and, often, inefficient procedure. Improving salt and frost tolerance may be achieved by direct gene transfer or through DNA mutation (Zhang et al. 2000). As a complementary approach to breeding activity, these methods provide an opportunity to improve a cultivar for a particular trait without disrupting the genotype or breaking desirable gene linkages. Among mutants lines released as commercial cultivars, $6 \%$ were reported to be tolerant to abiotic stress (Micke 1988).

Many plants accumulate compatible solutes, such as proline, in response to non-damaging levels of abiotic stress and these are thought to play a role in acclimation, reducing the effects of stress as it becomes more serious (Aspinall and Paleg 1981; Hasegawa et al. 2000). Proline levels can be manipulated in some species by in vitro selection in the presence of hydroxyproline (Van Swaaij et al. 1986, 1987; Dix 1993). Hydroxyproline is a toxic analogue of the amino acid proline and selection in the presence of hydroxyproline can lead to proline over-accumulation in cell lines and eventually to plants with heritable stress resistance (Riccardi et al. 1983; Dorffling et al. 1997). Selection for high proline has also been used as a biochemical marker for increased frost and salinity tolerance in conventional crop breeding programmes (Winkel 1989; Samaras et al. 1995; Delauney and Verma 1993; Silverira et al. 2003). Whilst proline it is not universally accepted as a successful selectant for all species some commercial proline over-accumulating cultivars of barley with improved frost resistance following hydroxyproline selection have been released (Tantau et al. 2004).

Cauliflower curd is known to be very responsive in tissue culture and a $10 \mathrm{~cm}$ diameter curd may carry up to 10 million meristems (Kieffer et al. 1998). Previous work has demonstrated that cauliflower is also responsive to mutagenesis and hydroxyproline selection resulting in proline overproducing in vitro lines (Deane et al. 1995). This earlier work was restricted by the culture methods available and more recently a protocol for the production of thousands of responsive meristematic lines of cauliflower in liquid culture has greatly enhanced the opportunity for developing selection procedures (Kieffer et al. 1995, 2001). The work here reports the assessment of abiotic stress resistance of cauliflower lines following mutagenesis and hydroxyproline selection using this new protocol. Preliminary results have already been reported (Fuller and Eed 2003) and the current paper reports the results of a larger population of lines and their stability following up to 15 routine sub-cultures and 2 years storage at low temperature $\left(5^{\circ} \mathrm{C}\right)$. With the larger volume of data generated it was possible to compare the responses of the lines both in vitro and in vivo and investigate correlations between cold, salt and hydroxyproline resistance and proline accumulation.

\section{Material and methods}

\section{Plant materials}

The January heading Roscoff F1 hybrid cauliflower Medaillon (courtesy of Elsoms Seeds Ltd) was grown in the field on the Seale-Hayne Estate, University of Plymouth, Devon, UK, according to good commercial practice (Anon 1982) and curds 
harvested and taken to the laboratory. In vitro microshoots were produced in liquid culture according to the technique described by Kieffer et al. $(1995,2001)$. This technique produced a high volume of single or double curd meristem explants in the size range $300-600 \mu \mathrm{m}$.

\section{Mutagenesis and selection}

Mutagenesis using $N$-nitroso- $N$-ethylurea (NEU) and $N$-Nitroso- $N$-methyl-urea (NMU) at 1 and $2.5 \mathrm{mM}$ for $90 \mathrm{~min}$ was carried out in liquid culture $24 \mathrm{~h}$ after preparation of the microshoots. Mutagens were removed by decanting and washing the microshoots three times in fresh liquid culture medium (Kieffer et al. 1995). All techniques were carried out according to the safe working practices established by McCabe et al. (1990).

Selection was carried out by the addition of $3 \mathrm{mM}$ hydroxyproline to the final liquid culture medium and incubating for 3 weeks. The experiment was repeated 7 times and approximately 1.5 million microshoots were exposed to the mutagens.

Green shoots surviving selection were removed from liquid medium and sub-cultured onto S23 solid culture medium (Kieffer et al. 1995) without hydroxyproline to develop into shoots. Shoots with obvious morphological abnormalities either died or were discarded. Selections were then subjected to a multiplication phase on S23M (S23 + Kinetin $2 \mathrm{mg} \mathrm{l}^{-1}$ and IBA $1 \mathrm{mg} \mathrm{l}^{-1}$ ) to produce clones of each selection, which were either rooted (Kieffer et al. 1995) and regenerated or put into cold storage $\left(5^{\circ} \mathrm{C}\right)$. A population of non-mutated/ selected control clones was also prepared from the same curd material. A number of clones were tested for their resistance to various stresses (Fuller and Eed 2003) and then maintained in vitro over a period of 2 years and reassessed for their resistance to various stresses.

Prior to any physiological assessments the in vitro cultures were sub-cultured to reinvigorate them. Existing plantlets were removed from culture pots and the apical meristem +1 emerging leaf, and stem sections with a single node, were cut from the plantlets and sub-cultured onto S23 medium. Where clones of lines existed then at least one clone of each line was sub-cultured to the multiplication medium $(\mathrm{S} 23 \mathrm{M})$ in order to build up clone numbers for experimentation. Cultures were maintained in a growth room at $23^{\circ} \mathrm{C}, 16$ photoperiod, $50 \mu \mathrm{mol}^{-1} \mathrm{~m}^{-2} \mathrm{~s}^{-1}$ and plantlets with 3-4 leaves were ready for testing for stress resistance 4-5 weeks after sub-culturing.

In vivo clones of most of the lines were obtained by weaning of plantlets 3-4 weeks after sub-culture. Plantlets were immersed in fungicide solution (Dithane $2 \mathrm{~g} \mathrm{l}^{-1}$ ) for $5 \mathrm{~min}$ prior to potting in trays filled with peat-based compost. The trays were placed in an incubator and shaded with a greenhouse shading material to encourage establishment and maintained in a glasshouse with a minimum temperature of $15^{\circ} \mathrm{C}$. After 2 3 weeks in vivo, roots had developed and the plants were removed from the incubator and repotted into $14 \mathrm{~cm}$ diameter pots and grown-on in the glasshouse. Plants typically had 3-4 leaves at the point of testing for stress resistance and were 6-7 weeks old post-weaning.

Analysis of salt resistance

Plants were screened for $\mathrm{NaCl}$ resistance using a leaf disc assay. One centimetre diameter leaf discs were cut from the leaves of plants of both in vitro and in vivo lines. Leaf discs from in vivo plants were first surface sterilized in $70 \%$ ethanol for $10 \mathrm{~s}$, followed by continuous shaking for $2 \mathrm{~min}$ in $10 \%$ commercial bleach solution (sodium hypochlorite) followed by three rinses in sterile distilled water. In vitro grown plants were used direct from culture vessels. Fifteen leaf discs were cut from each plant and placed in 3 replicate petridishes containing $20 \mathrm{ml}$ sterile liquid medium (M\&S salts at $4 \mathrm{~g} \mathrm{l}^{-1}$ ) supplemented with $\mathrm{NaCl}$ at concentrations of 350 and $550 \mathrm{mM}$ for in vitro and in vivo plantlets, respectively. Salt damage was assessed after 7 days using a five point score based on the percentage greenness of the leaf discs.

Analysis of hydroxyproline resistance

Leaf strips approximately $5 \mathrm{~mm}$ wide, were cut from the first and second leaves of both in vitro and in vivo plants. In vivo leaves were first surface sterilised (as for $\mathrm{NaCl}$ testing). Five leaf strips were placed in petri-dishes containing solid S23 
medium, supplemented with two concentrations of hydroxyproline ( 3 and $10 \mathrm{mM}$ for in vitro and in vivo shoots, respectively). After 4 weeks leaf strips were scored for resistance categorized by whether they displayed total, partial or no resistance evidenced by bleaching (disappearance of chlorophyll) and the percentage of leaf strips in each category was calculated.

\section{Estimation of free proline content}

In vitro shoot tips (apex plus emerging leaves and 1 emergent leaf) of the cauliflower lines were subcultured onto S23 medium supplemented with either $3 \mathrm{mM}$ hydroxyproline or $350 \mathrm{mM} \mathrm{NaCl}$ and grown for 4 weeks in the culture room. The first fully expanded leaf was then removed for determination of leaf proline content. Leaves were immediately frozen in a $-80^{\circ} \mathrm{C}$ freezer and then dried in a freeze drier to constant weight. Dried leaf $(0.04 \mathrm{~g})$, with $2 \mathrm{ml}$ ultra clean water, was finely ground in a pestle, taken into an eppendorf tube and centrifuged at $15,000 \mathrm{rpm}$ for $15 \mathrm{~min}$. Three aliquots of the resultant supernatant $(100 \mu \mathrm{l})$ were diluted to $1 \mathrm{ml}$ in 3 crimp top sample vials. HPLC was used to determine proline levels using a Dionex AAA-Direct Amino Acid Analyser System. A series of proline standards analysed by the acid ninhydrin method (Bates et al. 1973) and the Dionex system, found a good linear fit between the two methods (Fuller and Paisey personal communication) and concluded that the Dionex system is reliable, versatile, convenient and a suitable replacement for the more regularly used acid ninhydrin method. At first, 2 vials containing water were run on the Dionex to clean the column and stabilize the detector's reading. Then, 5 standard samples were run to obtain a calibration curve for all analyses. Afterwards, vials containing prepared samples were placed in the auto sampler and run overnight. Peaknet ${ }^{T M}$ software gave output curves from which the concentration of proline was integrated.

\section{Analysis of frost tolerance}

Frost tolerance was assessed using 6-7 week-old in vivo plants. Both non-acclimated (direct from glasshouse) and acclimated (after 14 days at $2^{\circ} \mathrm{C}$,
$8 \mathrm{~h}$ photoperiod $150 \mu \mathrm{mol}^{-1} \mathrm{~m}^{-2} \mathrm{~s}^{-1}$ irradiance) plants were assessed. Leaf discs, $1 \mathrm{~cm}$ in diameter, were cut from the newest fully expanded leaf avoiding the main veins and leaf margins. Twenty discs from each plant were randomly divided into sets of five discs and placed into glass test tubes. The tubes were chilled to $2^{\circ} \mathrm{C}$ and ice added to each tube to ensure ice nucleation. The tubes were placed in a Sanyo incubator programmed to run to $2,-3,-5$ and $-7^{\circ} \mathrm{C}$ (freezing rate $5^{\circ} \mathrm{C} \mathrm{h}^{-1}$ ) with a 2-hour hold at each temperature and duplicate samples removed at the end of each 2-hour hold. Tubes were allowed to thaw overnight at $4^{\circ} \mathrm{C}$ and then $12.5 \mathrm{ml}$ of distilled water was added to each tube. After 5-6 h incubation at $20^{\circ} \mathrm{C}$, the electrical conductivity of the water was determined using a Walden precision conductivity meter fitted with a platinum electrode (Fuller et al. 1989). The tubes were autoclaved at 15 psi for $5 \mathrm{~min}$ and cooled to $20^{\circ} \mathrm{C}$ and the conductivity re-measured. The degree of damage caused by freezing was expressed as a relative conductivity reading after correcting for the background conductivity of distilled water, Relative Conductivity percentage $(\mathrm{RC} \%)=$ Test reading/Final reading $\times$ 100. Analysis of variance (ANOVA) for all results was performed using Minitab, and means compared using the least significant difference (LSD) test.

\section{Heritability of resistance}

Populations of 15 control plants and the most resistant 15 selected plants were grown in separate glasshouses and allowed to flower. Crosspollination between individuals was encouraged by the introduction of blowflies to the glasshouses and F2 seed was collected. The two F2 populations were then tested for salt resistance and frost resistance (acclimated, tested at $-5,-7$ and $-9^{\circ} \mathrm{C}$ ) as described previously using 80 individual plants from each population.

\section{Results}

Salt resistance

Overall, leaf discs from both in vitro and in vivo plants exposed to $\mathrm{NaCl}$ treatment for 7 days 
Table 1 The effect of $\mathrm{NaCl}$ on the greenness of leaf discs of control and selected populations of cauliflower lines for in vitro and in vivo plants

\begin{tabular}{llllll}
\hline $\mathrm{NaCl}$ resistance & \multicolumn{2}{l}{ In vitro } & & \multicolumn{2}{l}{ In vivo } \\
\cline { 2 - 3 } & Absence of stress & $350 \mathrm{mM} \mathrm{NaCl}$ & & Absence of stress & $550 \mathrm{mM} \mathrm{NaCl}$ \\
\hline Control population mean $\pm \mathrm{SE}$ & $78.68 \mathrm{a} \pm 1.686$ & $17.44 \mathrm{~b} \pm 2.323$ & & $86.03 \mathrm{a} \pm 3.23$ & $17.49 \mathrm{~b} \pm 3.818$ \\
Selected population mean $\pm \mathrm{SE}$ & $86.72 \mathrm{a} \pm 1.561$ & $46.38 \mathrm{c} \pm 2.744$ & & $90.91 \mathrm{a} \pm 1.341$ & $55.08 \mathrm{c} \pm 2.959$
\end{tabular}

Means followed by the same letter are not significantly different

showed over $80 \%$ damage for the control population whilst the selected population showed a significant $(P<0.001)$ degree of resistance with less than $50 \%$ damage (Table 1$)$. Among the selected population there was a significant linear correlation between the in vitro and in vivo scores (Fig. 1). There was considerable variation in resistance demonstrated by individual lines and selections S2 and S17 showed a very high degree of resistance (Fig. 1).

\section{Hydroxyproline resistance}

Overall, leaf discs from both in vitro and in vivo plants exposed to hydroxyproline treatment showed a high degree of damage for the control population whilst the selected population showed a significant $(P<0.001)$ degree of resistance (Table 2). Among the selected population there was a significant positive curvi-linear relationship between the in vitro and in vivo scores (Fig. 2). As with $\mathrm{NaCl}$ resistance there was considerable variation between lines in the selected population (Fig. 2) with selected lines S2, S12, S6 and S1 showing high levels of resistance.

\section{Frost resistance}

Frost damage increased at progressively lower freezing test temperatures with $-7^{\circ} \mathrm{C}$ leading to high levels of damage. Acclimation significantly improved resistance in both populations at all test temperatures but the improvement was generally only small with a $1^{\circ} \mathrm{C}$ improvement due to acclimation. Significant linear correlations between test temperatures and between acclimated and non-acclimated responses of individuals demonstrated good reliability of the data (Fig. 3). The selected population (S) had significantly improved frost resistance at all test temperatures compared to the control under both acclimated and non-acclimated conditions (Fig. 4). In common with salinity and hydroxyproline stress the selected population demonstrated a wide range of frost resistance expression between lines with S2, S4, S1 and S21 showing the highest degree of frost resistance (Fig. 3).

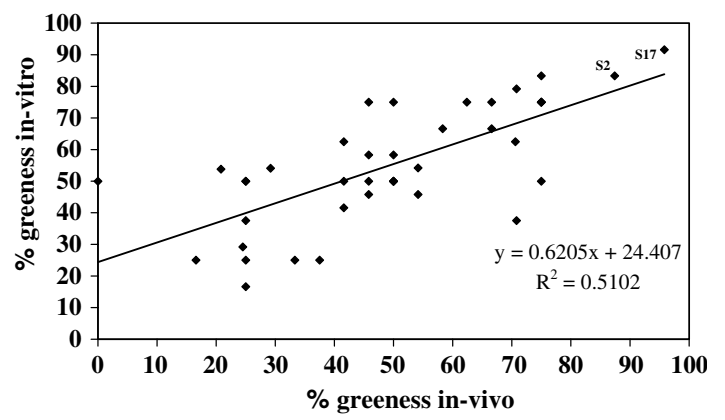

Fig. 1 The relationship between in vitro and in vivo responses to the salinity treatment in the selected population

Table 2 The effect of hydroxyproline (hyp) on the greenness of leaf strips of control and selected populations of cauliflower lines for in vitro and in vivo plants

\begin{tabular}{|c|c|c|c|c|}
\hline \multirow[t]{2}{*}{ Hydroxyproline resistance } & \multicolumn{2}{|l|}{ In vitro } & \multicolumn{2}{|l|}{ In vivo } \\
\hline & Absence of stress & 3 mM hyp & Absence of stress & 10 mM hyp \\
\hline Control population mean $\pm \mathrm{SE}$ & $73.62 \mathrm{a} \pm 2.342$ & $14.73 b \pm 1.656$ & $67.66 \mathrm{a} \pm 3.191$ & $12.02 b \pm 2.548$ \\
\hline Selected population mean $\pm \mathrm{SE}$ & $70.90 \mathrm{a} \pm 1.545$ & $27.55 c \pm 2.475$ & $73.04 \mathrm{a} \pm 1.791$ & $33.70 \mathrm{c} \pm 2.827$ \\
\hline
\end{tabular}

Means followed by the same letter are not significantly different 


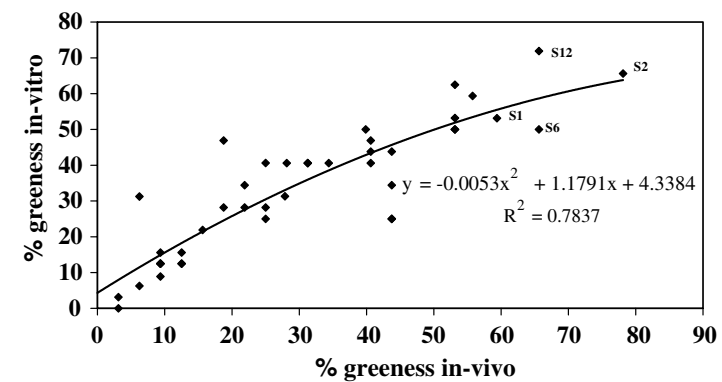

Fig. 2 The relationship between in vitro and in vivo responses to the hydroxyproline treatment in the selected population

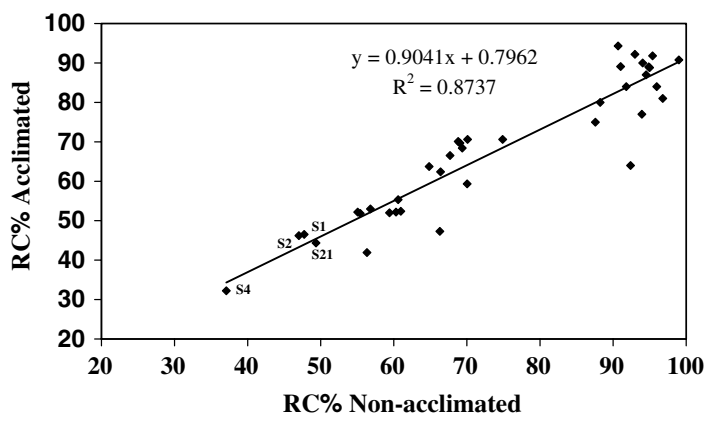

Fig. 3 Correlation between acclimated and non-acclimated frost hardiness expression at $-7^{\circ} \mathrm{C}$ for the selected population

\section{Proline levels}

Proline contents of the control population averaged less than $10 \mu \mathrm{g} \mathrm{mg}^{-1}$ under both non-stress and stress $(\mathrm{NaCl}$ and hydroxyproline) conditions whereas with the selected population proline content was significantly higher under non-stress conditions and much higher under stress conditions (Fig. 5). The greatest increase in proline content was when the selected population was stressed with $\mathrm{NaCl}$ with an overall average 17.5fold increase over the control population.

Within the selected population there was considerable variation in the levels of proline expressed under stress with the highest responding genotype ( $\mathrm{S} 17)$ expressing $926 \mu \mathrm{g} \mathrm{mg}^{-1}$ under $\mathrm{NaCl}$ stress, nearly 100 -fold increase over the control.

There was some correlation of proline levels with level of resistance although this was not strong $\left(R^{2}=0.3156\right.$ for $\mathrm{NaCl}$ resistance and

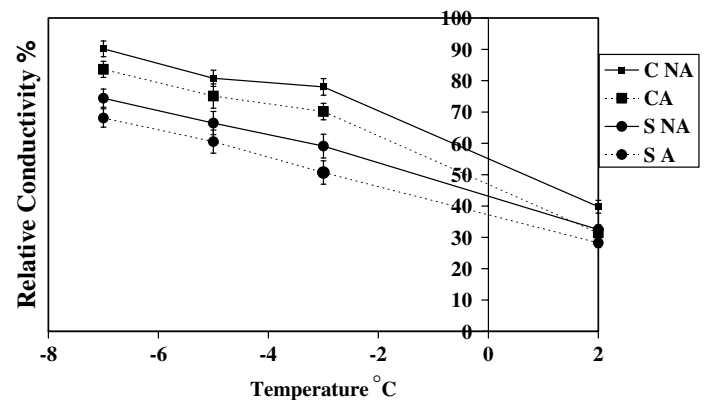

Fig. 4 Effect of acclimation (A) and non-acclimation (NA) on frost resistance of in vivo control (C) and selected (S) cauliflower populations

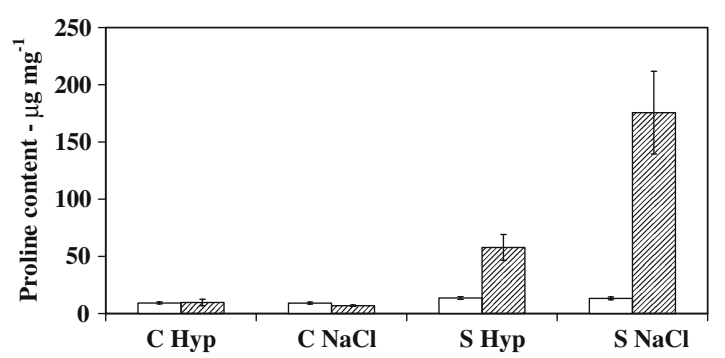

Fig. 5 Mean proline content of in vitro grown control (C) and selected populations of cauliflower without (unshaded columns) and with (shaded columns) treatment with $\mathrm{NaCl}$ $(350 \mathrm{mM})$ or hydroxyproline $(3 \mathrm{mM})$

$R^{2}=0.1128$ for hydroxyproline resistance) and despite some high proline/high resistant lines such as S2, S17, S9 and S1 (Figs. 6, 7) there were also some high proline low resistant lines particularly under hydroxyproline stress e.g. S7 and S82 (Fig. 7).

There was no overall correlation in the selected population under the 2 stresses (Fig. 8). Thus, lines which were high proline expressors under $\mathrm{NaCl}$ stress (S1, S17, S6, S21, S64) tended to be low expressors under hydroxyproline stress and vice versa (S7, S82, S4). Only 2 genotypes (S9, S31) showed very high proline under both stresses.

Summary of resistance data

The data for salt, hydroxyproline and frost resistance and proline accumulation were pooled to construct a profile of resistance expression for each line (not shown) and illustrated a variety of 


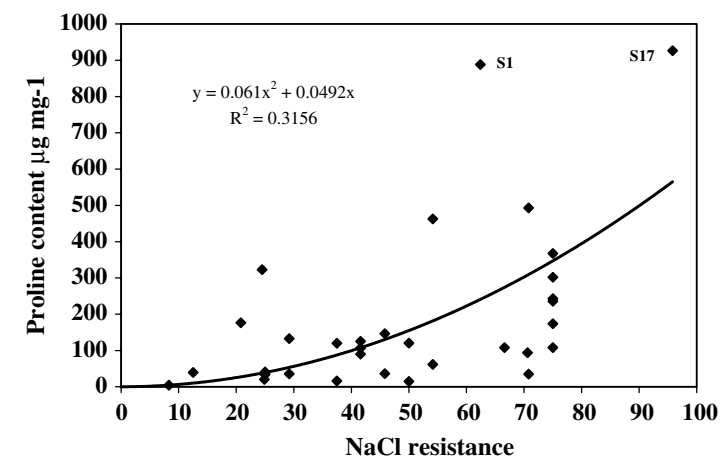

Fig. 6 Scattergram of in vitro $\mathrm{NaCl}$ resistance and proline content under $\mathrm{NaCl}$ stress for genotypes from the selected population

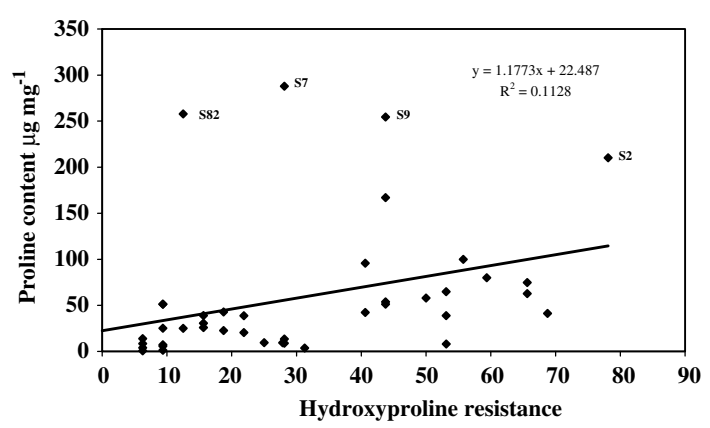

Fig. 7 Scattergram of relative in vitro hydroxyproline resistance and proline content under hydroxyproline stress for genotypes from the selected population

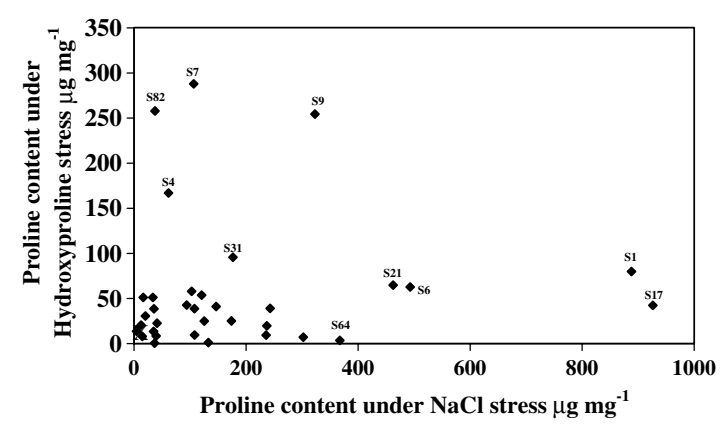

Fig. 8 Scattergram of proline content of selected genotypes under hydroxyproline stress versus under $\mathrm{NaCl}$ stress (control population response is marked by $\mathrm{X}$ )

complex effects. Only one of the lines (S2) showed high triple resistance (salt, hyp and frost) and high proline, five (S1, S17, S21, S100 and S101) showed high double resistance (salt and hyp) and high proline and six (S6, S31, S55, S64, S77, S125) showed high single resistance with high proline, while the remaining lines showed varying lower degrees of resistance to the 3 stresses and variable levels of proline. It appears therefore that some of lines selected against one stress factor were linked with resistance to others, but many lines showed no linkage between the stresses. A few of the selected lines (S46, S59 and S73) demonstrated little or no improved resistance to abiotic stress and no significant change in levels of proline and these must be classified as escapes, revertants or lines with epigenetic effects which are lost during sub-culturing. One line (S82) had high proline but no appreciable improvement in stress resistance.

The relative resistance measured here correlated well with that recorded earlier for 12 of the selections by Fuller and Eed (2003). This clearly demonstrated the stability of the induced mutations over time and through many in vitro subcultures and cold storage.

The responses of the selected lines were investigated by correlation for relationships between the responses to the 3 abiotic stresses. There was a weak negative correlation between frost and salt resistance, a week positive correlation between salt and hydroxyproline resistance and no relationship between frost and hydroxyproline (Fig. 9).

\section{Heritability of resistance}

Resistance to frost was demonstrated to be heritable with a significant reduction in mean frost damage at $-7^{\circ} \mathrm{C}$ and a significant shift in the population frequency distribution away from a normal distribution and with some individuals demonstrating a very high degree of resistance (Fig. 10). This distribution was similarly reflected in the results at $-9^{\circ} \mathrm{C}$ (not presented). This experiment was repeated to confirm these findings.

In contrast resistance to $\mathrm{NaCl}$ was shown not to be heritable with small but significant shift of the selected population as less resistant than the control population (Fig. 11). This experiment was repeated to confirm this finding. 
(a)

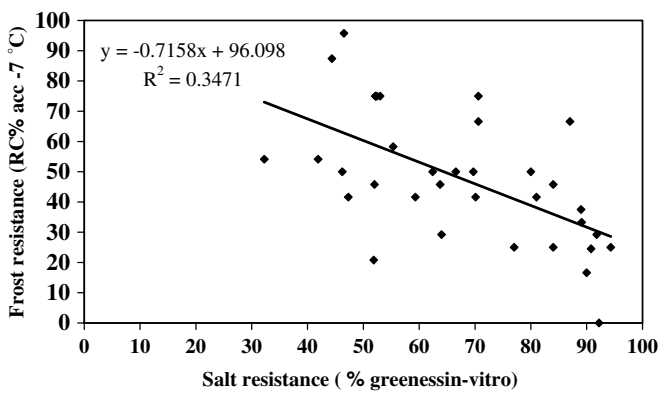

(b)

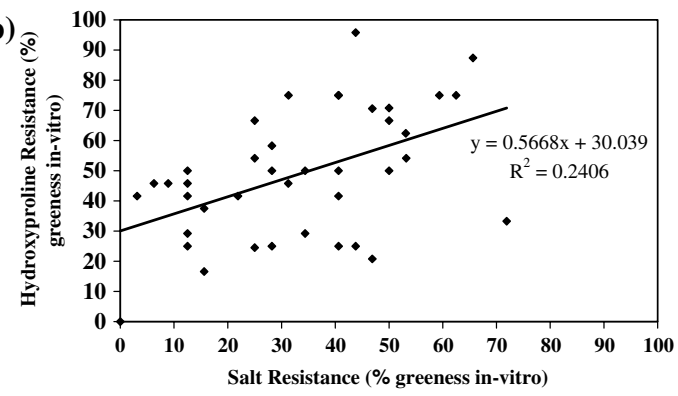

(c)

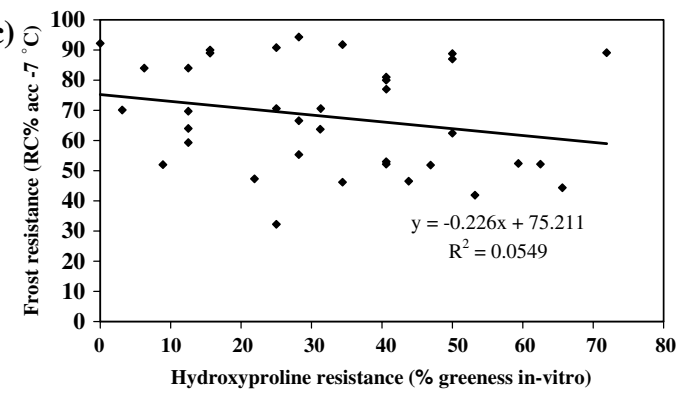

Fig. 9 The relative relationships of the selected lines to three abiotic stresses (a) frost resistance and salt resistance (b) hydroxyproline resistance and salt resistance (c) frost resistance and hydroxyproline resistance

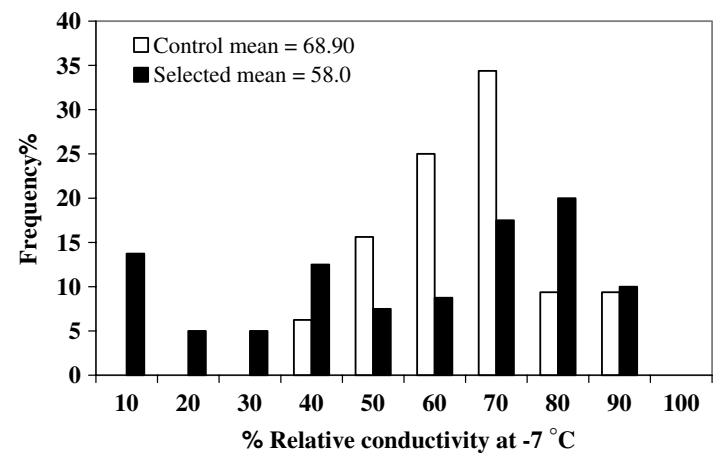

Fig. 10 Frequency histogram of frost resistance $(10=$ high, $90=$ low) of F2 control and selected populations

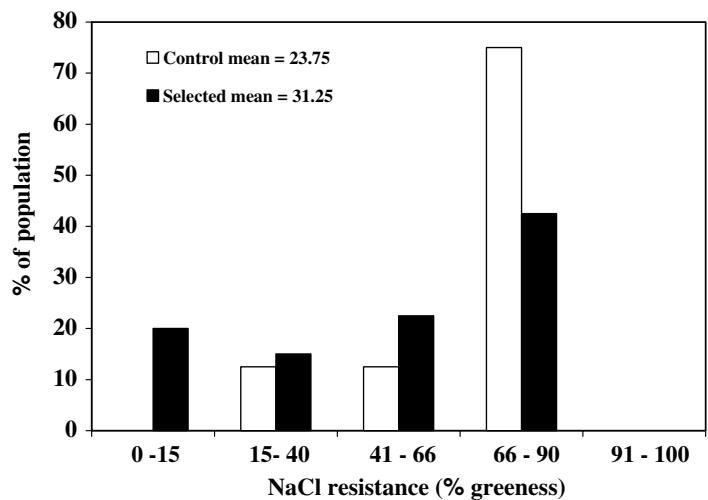

Fig. 11 Frequency histogram of salt resistance of control and selected populations

\section{Discussion}

The results presented here clearly show that the lines created by Eed (2002) by NEU and NMU mutagenesis retained abiotic stress resistance through many in vitro sub-cultures over a 2 3 year period and after prolonged storage at low temperature. Furthermore, when in vivo plants were weaned from these lines they demonstrated the same resistance as determined 2 years previously and this broadly correlated with the in vitro assessments of resistance indicating a stability of the traits. The short selection process with relatively high selection pressure that was used in this investigation clearly demonstrated that this kind of selection is useful in cauliflower to create genotypes resistant to abiotic stress as is the case in other Brassica species (Ashraf and Harris 2004; Ashraf and McNeilly 2004).

The heritability demonstrated for frost resistance was very encouraging and showed that the mutations were stable. Given that the original cultivar used in the investigation was an F1 hybrid it was unsurprising to see the genetic variation for frost resistance in the $\mathrm{F} 2$ generation of the control population but this variation remained normally distributed. The frequency distribution of the F2 mutant population gave an indication of segregation for the trait, which could be expected to polarise further in an F3 generation.

In contrast to the heritability for frost resistance, the heritability for salt resistance was disappointing and showed a tendency for segregation towards increased salt sensitivity. 
The experiments presented here give no definitive indication as to the mechanism(s) of abiotic resistance. The selection method of using hydroxyproline has assumed that resistance arises through an influence on the proline biosynthesis pathway such as decreased uptake of hydroxyproline (Widholm 1976) or preferential incorporation of the naturally occurring proline (Negrutiu et al. 1978) or a result of feedback insensitivity allowing proline to accumulate to levels at which it can successfully compete with hydroxyproline. The results clearly demonstrate that all of the selected mutant lines had higher proline levels than the controls when challenged with abiotic stress. Furthermore, nearly all of the mutant lines which demonstrated good resistance to stress all had high proline contents but there were exceptions-lines S42, S65 and S81 were all resistant to salt and/or frost but had relatively low proline. What is also clear from the results it that there is no absolute correlation of proline level and stress resistance. The range of mutants however provides abundant material to explore this question more thoroughly in the future. Many previous studies have shown that free proline accumulating cell lines show increased resistance to abiotic stress such as salt and freezing (Riccardi et al. 1983; Ashraf and McNeilly 2004; Kueh and Bright 1981; Dix et al. 1984; Tantau and Dorffling 1991; Dorffling et al. 1993) and the data presented here do not contradict these results but illustrate that resistance level is not necessarily proportional to the free proline content.

The evidence from this investigation suggests that whilst mutant lines of cauliflower can be created which demonstrate correlated resistance to more than one abiotic stress, there are equally lines which are clearly only resistant to a single stress. This together with the proline data suggests that many of the mutations that have been induced are likely to be in "downstream" operational genes rather than transcription factors. The findings also provide physiological evidence to support the molecular evidence that many genes are involved in the upregulation of resistance to abiotic stress (Zhu et al. 1997; Pearce 1999; Guy 2003; Vinocur and Altmann 2005). The metabolism of stress resistance in plants and the exact role of increased compatible solutes remains a major gap in our knowledge (Vinocur and Altmann 2005) and research efforts are being coordinated to investigate this in model species. The results of such efforts will then need to be corroborated in crop species and the existence of the range of mutants described in this paper will greatly assist future corroborative studies. In the meantime, agronomic evaluation of resistant mutants needs to be carried out to determine the stability of the mutant traits in the field.

It is concluded that elevated proline is not essential for improved resistance to abiotic stress in cauliflower, but where it does occur it does improve resistance.

\section{References}

Anon (1982) Cauliflower reference book 131. MAFF Grower Books, London 87 pp

Ashraf M, Harris P (2004) Potential biochemical indicators of salinity tolerance in plants. Plant Sci 166:3-16

Ashraf M, McNeilly T (2004) Salinity tolerance in Brassica oilseeds. Crit Rev Plant Sci 23:157-174

Aspinall D, Paleg L (1981) Proline accumulation: physiological aspects. In: Aspinall D, Paleg L (eds) The physiology and biochemistry of drought resistance in plants Academic Press, London pp 205-259

Bates L, Waldren R, Teare I (1973) Rapid determination of free proline for water stress studies. Plant Soil 39:263-268

Deane C, Fuller MP, Dix PJ (1995) Selection of hydroxyproline-resistant proline-accumulating mutant of cauliflower (Brassica oleracea var. botrytis). Euphytica 85:329-334

Delauney A, Verma D (1993) Proline biosynthesis and osmoregulation in plants. Plant J 4:215-223

Dix P (1993) Use of chemical and physical mutagens in vitro. In: Lindsey R (ed) Plant tissue culture manual. Kluwer Academic Press, pp 1-17

Dix P, McLysaght U, Plunkett A (1984) Salt stress: resistance mechanisms and in vitro selection procedures. In: Plant tissue culture and its agricultural applications. 41st Conf. Easter School Ser. Agric. Sci., Nottingham, UK pp 17-21

Dorffling K, Dorffling H, Lesselich G (1993) In vitro selection and regeneration of hydroxyproline-resistant lines of winter wheat with increased proline content and increased frost tolerance. J Plant Physiol 142:222-225

Dorffling K, Dorffling H, Lesselich G, Luke E, Zimmermann C, Melz G (1997) Heritable improvement of frost tolerance in winter wheat by in vitro selection of hydroxyproline resistant proline overproducing mutants. Euphytica 93:1-10 
Eed M (2002) The resistance to salt of brassica sps. and improved resistance by direct selection and mutagenesis. PhD thesis, The University of Plymouth, UK

Epstein E (1985) Salt tolerant crops: origin, development, and prospects of the concept. Plant Soil 89:187-198

Fuller MP, Grout BWW, Tapsell CR (1989) The pattern of frost hardening in leaves of winter Cauliflower (Brassica oleracea var. botrytis cv. Clause 30). Ann App Biol 115:161-170

Fuller MP, Eed M (2003) The development of multiple stress-resistant cauliflower using mutagenesis in conjunction with a microshoot tissue culture technique. Acta Hort 618:71-76

Guy CL (2003) Freezing tolerance of plants: current understanding and selected emerging concepts. Can J Bot 81:1216-1223

Hasegawa P, Bressan R, Zhu J, Bohnert H (2000) Plant cellular and molecular response to high salinity. Ann Rev Plant Physiol Plant Mol Biol 51:463-499

Kieffer ML, Fuller MP, Jellings AJ (1995) Rapid mass production of cauliflower propagules from fractionated and graded curd. Plant Sci 107:229-235

Kieffer ML, Fuller MP, Jellings AJ (1998) Explaining curd and spear geometry in broccoli, cauliflower and "romanesco": quantitative variation in activity of primary meristems. Planta 206:34-43

Kieffer ML, Simkins N, Fuller MP, Jellings AJ (2001) A cost effective protocol for in vitro mass propagation of cauliflower. Plant Sci 160:1015-1024

Kueh J, Bright S (1981) Proline accumulation in a barley mutant resistance to tran-4-hydroxy-L-proline. Planta 153:166-171

Mcabe P, Cseplo A, Timmons A, Dix P (1990) Selection of chloroplast mutants. In: Pollad J, Walker J (eds) Methods in molecular biology, vol 6, Plant cell and tissue culture. Humana Press, Clifton, New Jersey, pp 467-475

Micke A (1988) Genetic improvement of grain legumes using induced mutations: an overview. In: Improvement of genetic legume production using induced mutations. Proc. Workshop, Pullman, Washington, USA, July 1986. STI/PUB/766.Vienna, Austria: IAEA

Munns R, Rawson HM (1999) Effect of salinity on salt accumulation and reproductive development in the apical meristem of wheat and barley. Australian J Plant Physiol 26:459-465

Murashige T, Skoog F (1962) A revised medium for rapid growth and bioassays with tobacco tissue cultures. Physiol Plant 15:473-479

Negrutiu I, Jacobas M, Cattoir A (1978) Selection and characterization of resistant mutants to amino acid analogues in vitro plant cell culture. In: Abstracts of 4th international congress of plant tissue and cell culture, International Association for Plant Tissue Culture, Calary, Canada, p 138
Riccardi G, Cella R, Camerino G, Ciferri O (1983) Resistance to azetidine-2-carboxylic acid and sodium chloride tolerance in carrot cell cultures and Spirula platensis. Plant Cell Physiol 24:1073-1078

Pearce RS (1999) Molecular analysis of acclimation to cold. Plant Growth Reg 29:47-76

Samaras Y, Bresson RA, Csonka LN, Garcia-Rios M, Paina D'Urzo M, Rhodes D (1995) Proline accumulation during water deficit. In: Sambrook N (ed) Environment and plant metabolism flexibility and acclimation. BioScientific publishers, Oxford

Silverira J, Viegas RD, Da Rocha IM, Moreira AC, Moreira RD, Oliveira JT (2003) Proline accumulation and glutamine synthetase activity are increased by salt induced proteolysis in cashew leaves. J Plant Physiol 160:115-123

Tantau H, Dorffling K (1991) In vitro selection of hydroxyproline resistant cell lines of wheat (Triticum aestivum): accumulation of proline, decrease in osmotic potential and increase in frost tolerance. Physiol Plant 82:243-248

Tantau H, Balko C, Brettschneider B, Melz G, Dorffling K (2004) Improved frost tolerance and winter survival in winter barley (Hordeum vulgare L.) by in vitro selection of proline overaccumulating lines. Euphytica 139(1):19-32

Van Swaaij A, Nijdam H, Jacobsen E, Feenstra W (1986) Selection characterization and regeneration of hydroxyproline-resistant cell lines of Solanum tuberosum: tolerance to $\mathrm{NaCl}$ and freezing stress. Physiol Plant 68:359-366

Van Swaaij A, Nijdam H, Jacobsen E, Feenstra W (1987) Increased frost tolerance and amino acid content in leaves, tuber, leaf callus of regenerated hydroxyproline resistant potato clones. Euphytica 36:369-380

Vinocur B, Altman A (2005) Recent advances in engineering plant tolerance to abiotic stress: achievements and limitations. Current Opin Biotech 16:123-132

Wang W, Vinocur B, Shoseyour O, Altman A (2001) Biotechnology of plant osmotic stress tolerance: physiological and molecular considerations. Acta Hort 560:285-292

Widholm J (1976) Selection and characterisation of cultured carrot and tobacco cell resistant to lysine, methionine, and proline analogues. CJ Bot 54:1523-1529

Winkel A (1989) Breeding for drought tolerance in cereals. Vorträge Pflanzenzücht 16:357-368

Zhu J, Hasegawa P, Bressan R (1997) Molecular aspects of osmotic stress in plant. Crit Rev Plant Sci 16:253-277

Zhang J, Klueva N, Wang Z, Wu R, David-Ho T, Ngulen $H$ (2000) Genetic engineering for abiotic stress resistance in crop plants. In Vitro Cell Dev Biol Plant 36:108-114 